\title{
$\bullet$ IJCRR \\ Section: Healthcare \\ Evaluation of Synergistic Antioxidant Efficacy of Phyllanthus niruri Leaves and Sapindus mukorossi Fruits
} ISI Impact Factor (2019-20): 1.628 IC Value (2019): 90.81 $\operatorname{SJIF}(2020)=7.893$

(c) (i) (3) Copyright@IJCRR

\author{
Nazia Malik ${ }^{1}$, Rakesh Kumar Jat ${ }^{1}$, Darna Bhikshapathi ${ }^{2}$
}

'Institute of Pharmacy, Shri]agdish Prasad Jhabarmal Tibrewala University, Jhunjhunu, Rajasthan-3330o1, India; ${ }^{2}$ Teegala Ram Reddy College of Pharmacy, Pragathi Colony, Meerpet, Hyderabad-500097, Telangana, India.

\section{ABSTRACT}

Introduction: Numerous physiological and biochemical processes in the human body may produce oxygen-centred free radicals and other reactive oxygen species as by-products. Overproduction of such free radicals can cause oxidative damage to biomolecules, eventually leading to many chronic diseases, such as atherosclerosis, cancer, diabetes, ageing, and other degenerative diseases in humans. A traditional medicine prescription habitually contains various herbs or different parts, and these have a synergistic impact in effecting a cure or reducing toxicity.

Aims: The present study was aimed to investigate the effect of a combined extract of Phyllanthus niruri leaves and Sapindus mukorossi fruit on antioxidant activity by in vitro and in vivo methods.

Methods: In vitro antioxidant activity was performed by employing 2,2'-diphenyl-1-picrylhydrazyl (DPPH), Nitric oxide scavenging, reducing power, hydrogen peroxide scavenging, superoxide anion scavenging and $\beta$ - Carotene linoleate assays. In vivo antioxidant estimation has been performed using CCL4 and acetaminophen treated rats. Methanolic extract of Phyllanthus niruri leaves (MEPNL), aqueous extract of Sapindus mukorossi fruit (AESMF) and combined extract (MEPNL+AESMF) responses on antioxidant enzymes (Catalase, SOD and GSH) and lipid peroxidation were evaluated and compared.

Results: Combined extract (MEPNL+AESMF) at $400 \mathrm{mg} / \mathrm{kg}$ has exhibited significant antioxidant activity in in vitro methods by enhancing the scavenging of DPPH, NO, superoxide anion, increasing the reducing power and inhibition of bleaching of $\beta$-Carotene linoleic acid when compared to individual extracts (MEPNL \& AESMF). Lipid peroxidation was significantly decreased with combined extract than individual extracts. Catalase, SOD and Glutathione levels were remarkably elevated with the combination of extracts than individual extracts in $\mathrm{CCl} 4$ and Paracetamol treated rats.

Conclusions: Present study showed that the combined extract of Phyllanthus niruri leaves and Sapindus mukorossi fruit possess significant synergistic antioxidant activity than the individual leaf and fruit extracts demonstrated in vitro and in vivo methods. The synergistic effect of flavonoids, saponins and other antioxidant components in Phyllanthus niruri leaves and Sapindus mukorossi fruits may be responsible for enhanced antioxidant activity.

Key Words: Phyllanthus niruri, Sapindus mukorossi, Antioxidant, Synergistic, Flavonoids, Saponins

\section{INTRODUCTION}

These days, consideration is being centred on the investigation of the efficacy of plant in the customary medication since they are modest and have minimal side effects. ${ }^{1}$ Elective therapeutics dependent on nutraceutical treatment and phytotherapy have developed as new recuperating frameworks and rapidly and generally spread. ${ }^{2}$ Today, oxidative pressure has pulled in the consideration of analysts. An imbalance between free radicals and antioxidants prompts oxidative harm of proteins, fat, nucleic acids, and starches. ${ }^{3,4}$
Oxidation prevention agents have shielded the body from the unsafe impact of the free radicals. ${ }^{5}$ Endogenous antioxidant agents guard against the reactive oxygen species that are fortified by natural antioxidants that reinforce them and reestablish the ideal balance by neutralizing the ROS. ${ }^{6,7}$ Traditional medication has been utilized in various natural products and herbs to treat an assortment of sicknesses including dangerous tumours. ${ }^{8}$

Ongoing proof has exhibited that combination therapy could give more noteworthy helpful advantages to sicknesses,

\section{Corresponding Author:}

Nazia Malik, Institute of Pharmacy, Shri Jagdish Prasad Jhabarmal Tibrewala University, Jhunjhunu, Rajasthan-333001, India.

Mob: 7036333363; Email: naziamalik272020@gmail.com

ISSN: 2231-2196 (Print)

ISSN: 0975-5241 (Online)

Received: 24.10 .2020

Revised: 12.12 .2020

Accepted: 09.02.2021

Published: 05.07 .2021 
for example, AIDS, malignant growth, atherosclerosis and diabetes, all of which have complex aetiology and pathophysiology and therefore are hard to treat utilizing a single medication target approach. ${ }^{9}$ Various components in a herbal prescription exert a synergistic impact in such manners as following up on various targets or improving the solubility of active compounds, which constitute the pharmaceutical basis of traditional medicines. ${ }^{10}$

The leaves of the Phyllanthus niruri plant is reported to have multiple therapeutic properties such as anti-inflammatory, antipyretic and analgesic, antifungal, antimicrobial, antibacterial and antiparasitic, anti-cancer and Hepatoprotective activity. ${ }^{11}$ The fruit of the Sapindus mukorossi plant is reported to have expectorant, emetic, hepatoprotective and abortifacient effects. ${ }^{12}$

The active constituents present in both plants are flavonoids, tannins, saponins, alkaloids, Glycosides, phenolics and steroids. Since flavonoids and saponins have potent antioxidant properties. The present study was carried out to evaluate the antioxidant efficacy of combined extract of Phyllanthus niruri leaves and Sapindus mukorossi fruits by in vitro and in vivo methods and to compare the antioxidant potency of the combined extract with individual leaf and fruit extract and finally to establish the synergistic effect of combined extract over individual extracts.

\section{MATERIALS AND METHODS}

Collection and Authentication of Plant Material For the present study, the Phyllanthus niruri leaves and Sapindus mukorossi fruits were collected from the vicinity of the Meerpet, Saroornagar, Hyderabad. Sample specimens of Phyllanthus niruri leaves and Sapindus mukorossi fruits were deposited in a polythene bag. The sample specimens were kept in fresh condition by adding $2 \%$ formalin. The scientific name of the plant has been verified with http://www. theplantlist.org on $7^{\text {th }}$, March 2020. Plant materials were identified and authenticated by Dr. K. Venkata Ratnam, Rayalaseema University, Kurnool, Andhra Pradesh.

\section{Extraction}

The Phyllanthus niruri leaves and Sapindus mukorossi fruits were shade dried separately and mechanically reduced to a coarse powder. The weight of the coarse powders of Phyllanthus leaves and Sapindus fruits were found to be $1450 \mathrm{~g}$ and $1368 \mathrm{~g}$. The powders were subjected to hot continuous successive extraction in a Soxhlet apparatus with solvents in the increasing order of polarity using petroleum ether, ethyl acetate, acetone, methanol and water under controlled temperature $\left(50-60^{\circ} \mathrm{C}\right)$. The extracts thus obtained were concentrated in a vacuum rotary evaporator and extracts were kept in desiccators for further use.

\section{Phytochemical screening}

Phytochemical qualitative analysis was performed by subjecting the crude extracts for identification tests to detect the presence of flavonoids, glycosides, alkaloids, carbohydrates, fixed oils, tannins, phytosterols, proteins, amino acids, lignins, phenolic compounds, saponins, gums \& mucilages. ${ }^{13}$

Methanolic extract of Phyllanthus niruri leaves (MEPNL) and aqueous extract of Sapindus mukorossi fruits (AESMF) were found to possess a significant number of active constituents and are selected for antioxidant activity. The combined extract was prepared by mixing the extracts of Phyllanthus niruri leaves and Sapindus mukorossi fruits in an equal ratio $(1: 1)$.

\section{Animals}

Wistar rats (180-200 g) were procured from Sainath agencies, Musheerabad, Hyderabad (282/99/CPCSEA) and housed in the animal facility of the institution. After randomly dividing the animals into different groups, the rats were accustomed for one month before the initiation of the experiment. Animals were caged in polypropylene cages and preserved under standard environmental conditions such as temperature $\left(26 \pm 2^{\circ} \mathrm{C}\right)$, relative humidity (45-55\%) and $12 \mathrm{hr}$ dark/light cycle. The animals were fed with a rat pellet diet (Golden Mohur Lipton India Ltd.) and water ad libitum.

\section{CPCSEA / IAEC Approval for Animal Studies}

The study protocol was approved by the institutional ethical committee with reference no: 1447/PO/Re/S/11/12/A.

\section{Experimental methods}

In vitro antioxidant activity of methanolic extract of Phyllanthus niruri leaves (MEPNL), aqueous extract of Sapindus mukorossi fruits (AESMF) and combined extract (MEPNL+AESMF)

1. DPPH assay: To $1 \mathrm{ml}$ of DPPH $(0.1 \mathrm{mM}$ solution) in methanol add $3 \mathrm{ml}$ of different concentrations of MEPNL, AESMF \& combined extract (MEPNL+AESMF) $(20,40,60,80 \& 100 \mu \mathrm{g} / \mathrm{ml})$. The reference standard used is ascorbic acid $(100 \mu \mathrm{g} / \mathrm{ml})$. All the experiments were reproduced threefold. The percentage suppression (inhibition) was evaluated by using a formula

$$
\% \text { Inhibition }=\frac{\text { Control Abs }- \text { Absorbance of sample }}{\text { Absorbance of control }} \times 100
$$

2. Nitric oxide scavenging assay ${ }^{14}$ : Sodium nitroprusside $(2 \mathrm{ml})$ in $\mathrm{pH} 7.4$ phosphate buffer is combined with $0.5 \mathrm{ml}$ of different strengths of MEPNL, AESMF $\&$ combined extract (MEPNL+AESMF) ranging from $5-100 \mu \mathrm{g} / \mathrm{ml}$ dissolved in methanol. The mixtures are kept for incubator at $250 \mathrm{C}$ at room temperature 
for 150 minutes. Control consists of the same reaction mixture excluding MEPNL and AESMF. Griess reagent $(0.5 \mathrm{ml})$ was added after incubation of individual sample (up to $0.5 \mathrm{ml}$ ) and further incubated for 30 minutes. The absorbance of chromophore was recorded at wavelength $546 \mathrm{~nm}$. This experiment was executed threefold and the $\%$ inhibition was evaluated with the formula

$\%$ Inhibition $=\frac{\text { Control Abs }- \text { Sample Abs }}{\text { Control Abs }} \times 100$

3. Reducing Power assay: The reducing power potency of the MEPNL, AESMF and combined extract (MEPNL+AESMF) was assessed by Oyaizu method $^{15}$. Distilled water $(1 \mathrm{ml})$ possessing different strengths of MEPNL, AESMF and combined extract (MEPNL+AESMF) $(20-100 \mu \mathrm{g} / \mathrm{ml})$ were combined with phosphate buffer ( $\mathrm{pH} 6.6,2.5 \mathrm{ml}, 0.2 \mathrm{M}$ ), 1\% $\mathrm{K} 3[\mathrm{Fe}(\mathrm{CN}) 6](2.5 \mathrm{ml})$ and incubated at $500 \mathrm{C}$ for 20 minutes. After incubation, a part of TCA $(2.5 \mathrm{ml}) 10 \%$ was combined and solutions were centrifuged for 10 minutes $(3000 \mathrm{rpm})$. Supernatant solutions i.e the above layers were combined with distilled water $(2.5$ $\mathrm{ml})$ and $\mathrm{FeCl} 3(0.5 \mathrm{ml})(0.1 \%)$ and the absorbance has been interpreted at $700 \mathrm{~nm}$. Sodium metabisulphite was considered as the reference standard. The test was conducted in triplicate. Absorbance is directly proportional to the reducing power. A rise in the reaction mixture absorbance suggests enhancement in reducing power. Percentage rise in absorbance was determined by the formula

$\%$ Increase in reducing power $=\frac{\mathrm{OD} \text { of test }-\mathrm{OD} \text { of control }}{\mathrm{OD} \text { of control }} \times 100$

4. Hydrogen peroxide Scavenging Assay: ${ }^{16,17}$ Different concentrations of MEPNL, AESMF \& combined extract (MEPNL+AESMF) (50-250 $\mu \mathrm{g} / \mathrm{ml})$ was combined with hydrogen peroxide $(2 \mathrm{ml}$ of $20 \mathrm{mM}$ ) in PBS (pH7.5) and incubated for 10 minutes. The absorbance was recorded at $230 \mathrm{~nm}$ against PBS blank. The experiment was carried out in a triplet. Data were represented as \% inhibition. For reference standard ascorbic acid was used.

$\%$ Increase in absorbance $=\frac{\text { Control OD-Test OD }}{\text { OD of Control }} \times 100$

5. Superoxide Anion scavenging assay: $100 \mu 1$ riboflavin $(2 \mu \mathrm{m}), 200 \mu \mathrm{l}$ methionine $(13 \mathrm{mM}), 200 \mu \mathrm{l}$ EDTA (100 $\mu \mathrm{m}), 100 \mu \mathrm{l}$ of $75 \mu \mathrm{m} \mathrm{NBT}$ and MEPNL, AESMF $\&$ combined extract (MEPNL+AESMF) (100, 200, 300,400 and $500 \mu \mathrm{g} / \mathrm{ml}$ ) were combined and further dissolved with sodium phosphate buffer (up to $3 \mathrm{ml}$ ). Generation of formazon was accompanied by reading the absorbance under $560 \mathrm{~nm}$ after illumination for 10 minutes by using a lamp of fluorescent light. The solution was kept at dark in similar tubes act as blanks. Percentage suppression has been determined by utilizing the formula.

$\%$ Inhibition $=\frac{\text { Control Abs }- \text { Abs. of sample }}{\text { Abs. of control }} \times 100$

6. $\beta$-Carotene Linoleate assay: ${ }^{18} 2 \mathrm{mg}$ of B-Carotene is liquefied in $10 \mathrm{ml} \mathrm{CHCl}_{3}$ and the former solution $(2 \mathrm{ml})$ was passed into $100 \mathrm{ml} \mathrm{R}$.B flask. $\mathrm{CHCl}_{3}$ was eliminated using vacuum and a further $40 \mathrm{mg}$ of linoleic acid, $400 \mathrm{mg}$ tween 40 , followed by $100 \mathrm{ml}$ distilled $\mathrm{H} 2 \mathrm{O}$ were added. The above intermixture $(4.8 \mathrm{ml})$ is supplemented to the tubes possessing MEPNL, AESMF and combined extract (MEPNL+AESMF) $(100,200$, $300,400 \& 500 \mu \mathrm{g} / \mathrm{ml})$ in $2 \mathrm{ml}$, the absorbance of the zero period was calculated using $470 \mathrm{~nm}$. The tubes were incubated at $500 \mathrm{C}$ for a period of $2 \mathrm{hr}$ and further recorded the absorbance. A $\beta$-carotene blank was prepared \& recorded its absorbance. The similar technique has been reciprocated using BHT.

Antioxidant potency $=(\beta$-carotene amount after $2 \mathrm{hr}$ of assay/ initial $\beta$-Carotene content) x100

\section{Single-dose oral acute toxicity for one week with gross behavioural study ${ }^{19,20}$}

The Acute toxicity evaluation of MEPNL and AESMF were performed based on OECD guidelines 423 by using mice and fixed-dose studies were selected where the limit dose is $2000 \mathrm{mg} / \mathrm{kg}$ ( Figure 1).

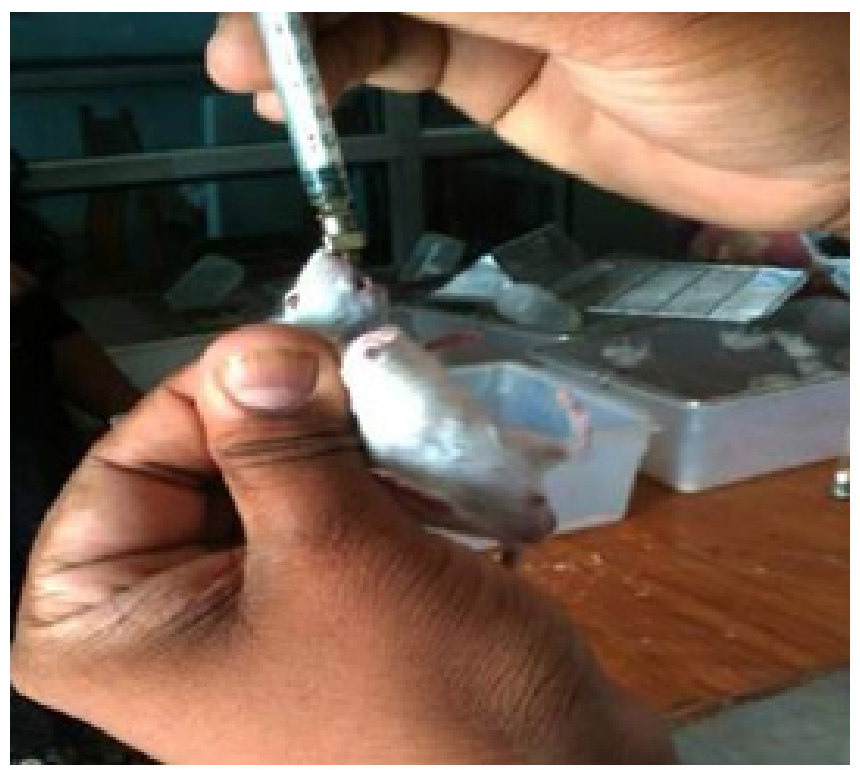

Figure 1: Single-dose oral acute toxicity studies of MEPNL and $A E S M F$ in albino mice. 
In vivo antioxidant studies of MEPNL, AESMF and combined extract (MEPNL+AESMF)

1). Carbon tetrachloride $\left(\mathrm{CCl}_{4}\right)$ intoxicated rats: ${ }^{21}$ Rats were separated into 8 groups consisting of 6 rats in each group

I: Untreated control ( $1 \%$ liquid paraffin $1 \mathrm{ml} / \mathrm{kg}$ s.c as vehicle) (-ve control)

II: Hepatotoxin control (+ve control) (vehicle for only 7 days (s.c) subsequently $1 \mathrm{ml} / \mathrm{kg}$ b.w $\mathrm{CCl}_{4}$ :liq.paraffin (1:1) s.c on day 7)

III: Standard group (Silymarin $100 \mathrm{mg} / \mathrm{kg}$ ) one time daily for 7 days and then $\mathrm{CCl}_{4}$ : Liq. paraffin (1:1) $1 \mathrm{ml} / \mathrm{kg}$ s.c on 7th day.

IV: MEPNL (200 mg/kg) every day for one week orally and then $\mathrm{CCl}_{4}$ :liq.paraffin $(1: 1)$ s.c on day 7 .

V: MEPNL $(400 \mathrm{mg} / \mathrm{kg}$ ) every day for one week orally and then $1 \mathrm{ml} / \mathrm{kg} \mathrm{CCl}$ :liq.paraffin (1:1) s.c on 7 th day.

VI: AESMF $(200 \mathrm{mg} / \mathrm{kg})$ every day for one week orally and then CCl4:liq.paraffin (1:1) s.c on day 7

VII:AESMF (400 mg/kg) every day for one week orally and then $1 \mathrm{ml} / \mathrm{kg} \mathrm{CCl} 4$ :liq.paraffin (1:1) s.c on 7th day.

VIII: Combined extract (MEPNL+ AESMF) (400 mg/ $\mathrm{kg}$ ) every day for one week orally and then $1 \mathrm{ml} / \mathrm{kg}$ $\mathrm{CCl}_{4}$ :liq.paraffin (1:1) s.c on $7^{\text {th }}$ day.

2) Paracetamol intoxicated rats: ${ }^{22}$ Rats were bifurcated in to 8 groups comprising of 6 rats in each group.

I: Untreated control (-ve control) (acacia suspension 2\% $\mathrm{w} / \mathrm{v}$ in distilled water)

II: Treated toxic control (+ve control) (1 week vehicle + paracetamol $2 \mathrm{~g} / \mathrm{kg}$ orally on 5 th day)

III: Standard (Silymarin $100 \mathrm{mg} / \mathrm{kg}$ every day for 1 week orally $+2 \mathrm{~g} / \mathrm{kg}$ acetaminophen orally on 5 thday).

IV: MEPNL (200 mg/kg dose every day for 1 week $+2 \mathrm{~g}$ / $\mathrm{kg}$ acetaminophen on 5 th day).

V: MEPNL ( $400 \mathrm{mg} / \mathrm{kg}$ dose every day for 7 days orally $+2 \mathrm{~g} / \mathrm{kg}$ acetaminophen orally on 5 th day).

VI: AESMF (200 mg/kg dose every day for 1 week $+2 \mathrm{~g} /$ $\mathrm{kg}$ acetaminophen on 5 th day).

VII:AESMF $(400 \mathrm{mg} / \mathrm{kg}$ dose every day for 1 week $+2 \mathrm{~g} /$ $\mathrm{kg}$ acetaminophen on 5th day).

VIII: Combined extract (MEPNL+ AESMF) [(400 mg/kg) dose every day for 1 week $+2 \mathrm{~g} / \mathrm{kg}$ acetaminophen on 5th day].

\section{Liver Isolation}

The rats were killed using mild ether anaesthesia on day 8th for liver isolation. The isolated liver was completely cleansed with an ice cold solution of saline. The liver weight was recorded after blotting it in pads of filter paper. A liver homogenate was made which was utilized for further in vivo antioxidant analysis.

Statistical analysis: The findings were exhibited as Mean \pm S.E.M by using one way ANOVA subsequently using Tukeykramer comparison analysis. The results were regarded to be statistically significant when $\mathrm{p}<0.05$.

\section{RESULTS}

\section{Phytochemical investigations}

Preliminary phytochemical investigation of MEPNL and AESMF indicates the presence of flavonoids, saponins, alkaloids, tannins, carbohydrates, glycosides, fixed oils, phenolics, fats and lignins. Methanolic extract of Phyllanthus niruri leaves and aqueous extract of Sapindus mukorossi fruit were selected for in vitro and in vivo Pharmacological activities. Flavonoids are detected more in methanolic extract of Phyllanthus niruri leaves and flavonoids are reported to have antioxidant and organ protective properties. Saponins are detected with greater clarity in the aqueous extract of Sapindus mukorossi fruit and saponins are reported to be responsible for the antioxidant and antistress activity.

\section{Preparation of combined extract of Phyllanthus niruri leaves and Sapindus mukorossi fruit}

Both the leaf and fruit extracts were combined in equal ratios (1:1) i.e 5 gms of Phyllanthus niruri leaf extract with 5 gms of Sapindus mukorossi fruit extract.

\section{In vitro antioxidant studies of MEPNL, AESMF and com- bined extract (MEPNL+AESMF)}

1. DPPH assay: In this investigation, MEPNL, AESMF and combined extract (MEPNL+AESMF) have exhibited a doserelated rise in DPPH radical scavenging action. L-Ascorbic acid (in a dose of $100 \mu \mathrm{g}$ ) has exhibited $78.52 \%$ scavenging action. However MEPNL and AESMF at $100 \mu \mathrm{g} / \mathrm{ml}$ have shown scavenging activity i.e $19.31 \%$ \& $45.80 \%$. Combined extract (MEPNL+AESMF) has shown highly significant DPPH scavenging activity to an extent of $90.23 \%$ which is greater than the individual plant extracts and also significant than standard ascorbic acid. The results are graphically represented in figure 2 .

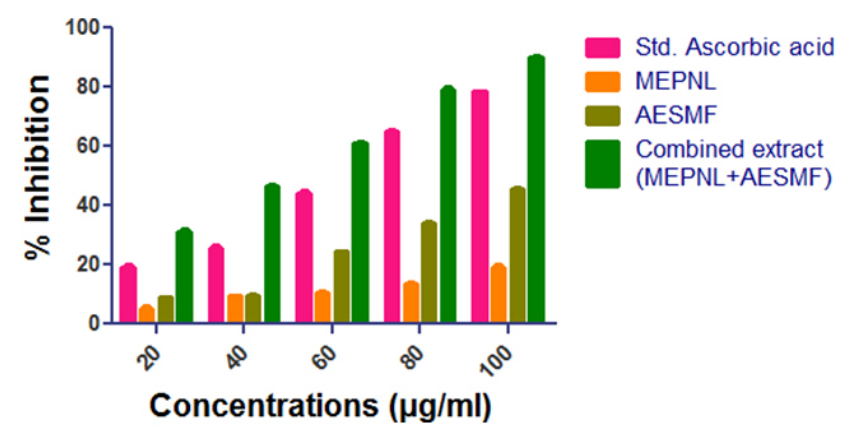

Figure 2: DPPH radical scavenging activity of MEPNL, AESMF and Combined Extract (MEPNL+ AESMF).

2. NO radical scavenging activity: MEPNL, AESMF and combined extract have shown a considerable dose-related rise 
in the NO anion scavenging action. Ascorbic acid $(100 \mu \mathrm{g})$ has exhibited $74.382 \%$ potency. MEPNL and AESMF (100 $\mu \mathrm{g})$ has shown significant scavenging activity i.e $24.934 \%$ \& 48.588\%. Combined extract (MEPNL+AESMF) has exhibited greater NO anion scavenging action to an extent of $57.228 \%$ which is higher than the NO scavenging activity of individual extracts. The results are graphically shown in figure 3 .

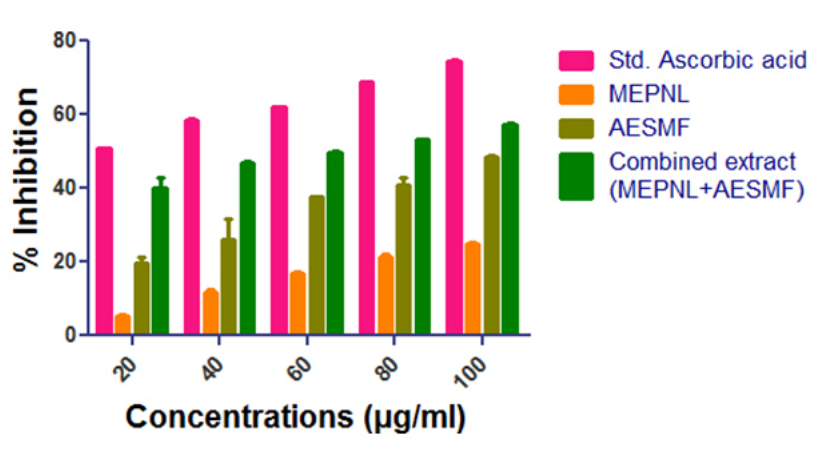

Figure 3: Nitric oxide scavenging activity of MEPNL, AESMF and Combined extract (MEPNL+AESMF).

3. Reducing Power assay: From the above assay, it is evident that MEPNL, AESMF and Combined extract (MEPNL+AESMF) have exhibited a dose-dependent increase in the reducing activity. Standard sodium metabisulphite $(100 \mu \mathrm{g})$ has shown $92.48 \%$ activity. MEPNL and AESMF at 20, 40, $60 \& 80 \mu \mathrm{g}$ have exhibited a steady increase in reducing activity, but a significant increase in the activity was observed with MEPNL \& AESMF at a concentration of $100 \mu \mathrm{g}(69.29 \& 74.53 \%)$. Combined extract (MEPNL+AESMF) has exhibited a greater increase in reducing power action to an extent of $84.57 \%$ which is higher than the reducing power activity of individual extracts. The results are tabulated in Table No. 3 and graphically illustrated in figure 4.

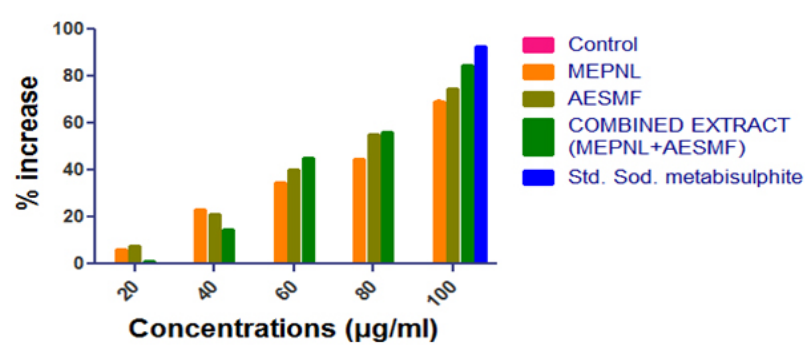

Figure 4: Reducing power activity of MEPNL, AESMF and Combined extract (MEPNL+AESMF).

4. Hydrogen peroxide scavenging activity: MEPNL, AESMF and Combined extract (MEPNL+AESMF) exhib- ited dose-dependent $\mathrm{H} 2 \mathrm{O} 2$ scavenging activity. Standard ascorbic acid $(250 \mu \mathrm{g})$ has shown $71.774 \%$ activity. MEPNL and AESMF exhibited maximum activity at 250 $\mu \mathrm{g}(29.201 \%$ and $41.007 \%$ ) with IC50 values of $405.67 \mu \mathrm{g} \& 345.47 \mu \mathrm{g}$. Combined extract (MEPNL+AESMF) at dose $250 \mu \mathrm{g} / \mathrm{ml} \mathrm{ex-}$ hibited a synergistic $\mathrm{H}_{2} \mathrm{O}_{2}$ scavenging activity action to an extent of $51.613 \%$ which is higher than the $\mathrm{H}_{2} \mathrm{O}_{2}$ scavenging activity of individual extracts.

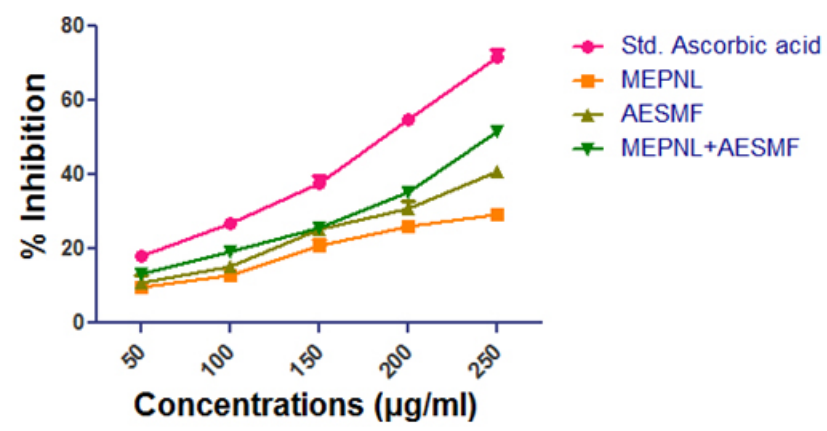

Figure 5: Hydrogen peroxide scavenging activity of MEPNL, AESMF and Combined extract (MEPNL+AESMF).

5. Superoxide anion scavenging activity: MEPNL and AESMF have shown moderate scavenging activity i.e $45.332 \%$ \& 51.304\% at $500 \mu \mathrm{g} / \mathrm{ml}$. Combined extract (MEPNL+AESMF) at dose 500mg. (Figure 5) $\mu \mathrm{g} / \mathrm{ml}$ exhibited a synergistic superoxide anion scavenging activity to an extent of $61.929 \%$ which is greater than the superoxide anion scavenging activity of individual extracts. The results are illustrated in figure $\mathbf{6}$.

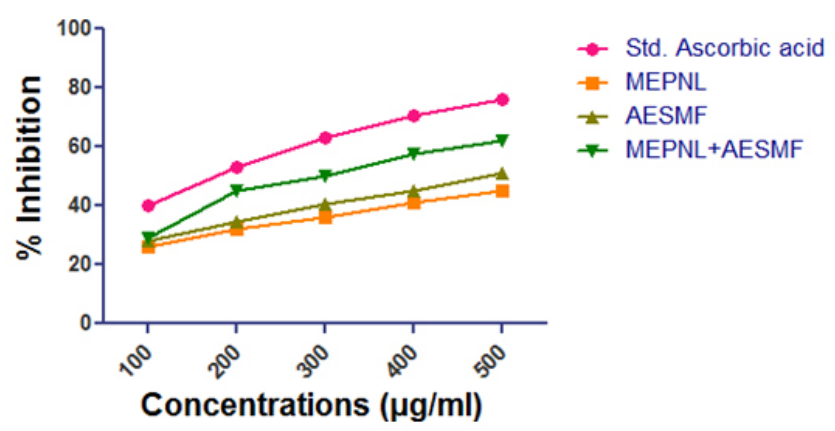

Figure 6: Superoxide anion scavenging activity of MEPNL, AESMF and Combined extract (MEPNL+AESMF).

6. Antioxidant activity in $\beta$-Carotene linoleate system: BHT $(500 \mu \mathrm{g})$ has exhibited $74.653 \%$ activity. MEPNL and AESMF $(500 \mu \mathrm{g})$ have shown mild scavenging activity i.e $11.607 \%$ \& $17.956 \%$ compared to standard BHT. Combined extract (MEPNL+AESMF) at dose $500 \mu \mathrm{g} / \mathrm{ml}$ exhibited a significant increase in scavenging activity to an extent of $60.317 \%$ which is much greater than the scavenging activity of individual extracts ( Figure 7). 


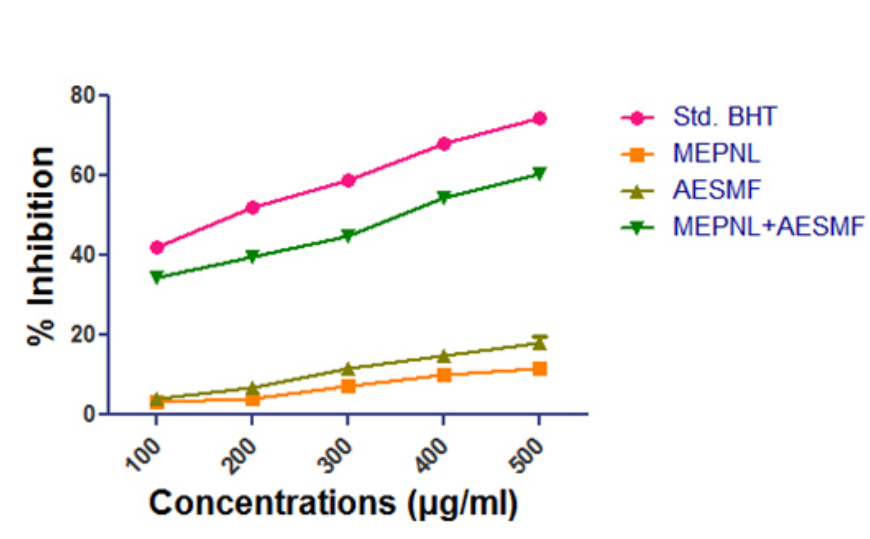

Figure 7: Antioxidant activity of MEPNL, AESMF and Combined extract (MEPNL+AESMF) in $\beta$-Carotene linolate system Acute toxicity studies of MEPNL and AESMF.

The methanolic extract of Phyllanthus niruri leaves (MEPNL) and aqueous extract Sapindus mukorossi fruit (AESMF) were administered to mice at doses 5, 50, 300 \& $2000 \mathrm{mg} / \mathrm{kg}$ with an oral syringe did not display any symptoms of toxicity. The rats were examined for two weeks, twice a day has not exhibited toxic signs. Hence oral LD50 of MEPNL \& AESMF was finalized to surpass $2000 \mathrm{mg} / \mathrm{kg}$. Therefore $2000 \mathrm{mg} / \mathrm{kg}$ was regarded as the safest higher dose for methanolic extract Phyllanthus niruri leaves and aqueous extract Sapindus mukorossi fruit and 1/10th i.e $200 \mathrm{mg} / \mathrm{kg}$ (lower dose) and 1/5th of $2000 \mathrm{mg} / \mathrm{kg}$ i.e $400 \mathrm{mg} / \mathrm{kg}$ (higher dose) of MEPNL and AESMF were preferred for the further pharmacologicalstudies.

\section{In vivo antioxidant activity of MEPNL, AESMF and Combined extract (MEPNL+AESMF) in $\mathrm{CCl}_{4}$ and Par- acetamol intoxicated rats}

LPO was considerably reduced by MEPNL and AESMF at $200 \& 400 \mathrm{mg} / \mathrm{kg}$ in $\mathrm{CCl}_{4}$ and Paracetamol intoxicated rats. However maximum reduction of LPO was seen with combined extract at $400 \mathrm{mg} / \mathrm{kg}$ in $\mathrm{CCl}_{4}$ and Paracetamol intoxicated rats. Proportions of catalase are considerably enhanced with MEPNL and AESMF at $400 \mathrm{mg} / \mathrm{kg}$ in $\mathrm{CCl}_{4}$ and Paracetamol intoxicated rats, whereas the degree of catalase was not significantly increased with $200 \mathrm{mg} / \mathrm{kg}$. Combined extract at $400 \mathrm{mg} / \mathrm{kg}$ exhibited a significant enhancement of catalase activity than individual extracts. SOD and glutathione levels were remarkably increased with MEPNL and AESMF at $400 \mathrm{mg} / \mathrm{kg}$. Combined extract (MEPNL + AESMF) at $400 \mathrm{mg} / \mathrm{kg}$ exhibited a significant increase in SOD and glutathione levels than MEPNL and AESMF in $\mathrm{CCl}_{4}$ and Paracetamol intoxicated rats. Silymarin exhibited a considerable effect on all the parameters at $100 \mathrm{mg} / \mathrm{kg}$ shown in Figure 8,9,10 and 11.
Lipid Peroxidation

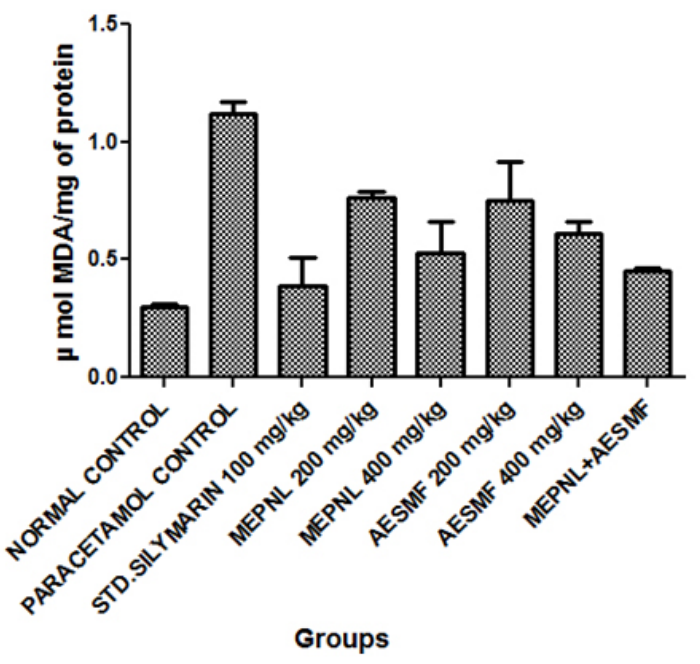

Figure 8: Effect of MEPNL, AESMF and Combined extract (MEPNL+AESMF) on LPO in acetaminophen treated rats.

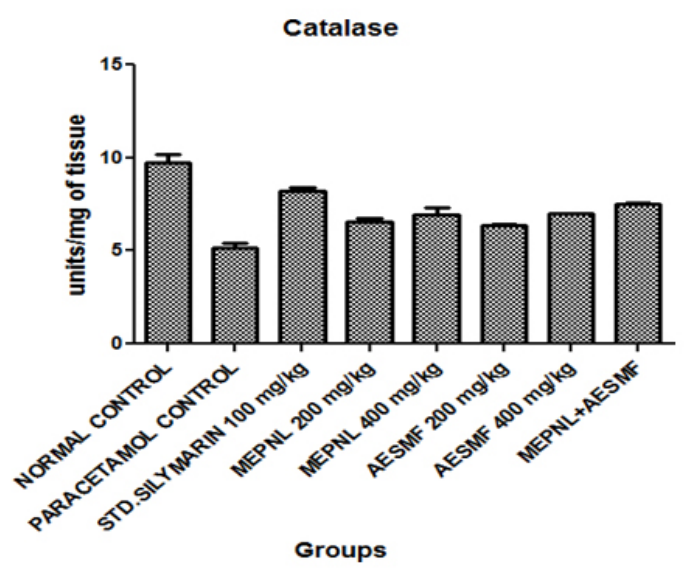

Figure 9: Effect of MEPNL, AESMF and Combined extract (MEPNL+AESMF) on catalase in acetaminophen treated rats.

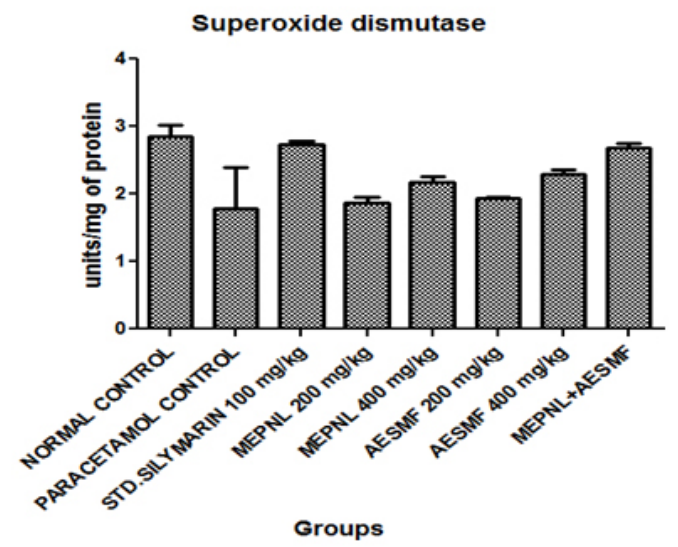

Figure 10: Effect of MEPNL, AESMF and Combined extract (MEPNL+AESMF) on SOD in acetaminophen treated rats. 


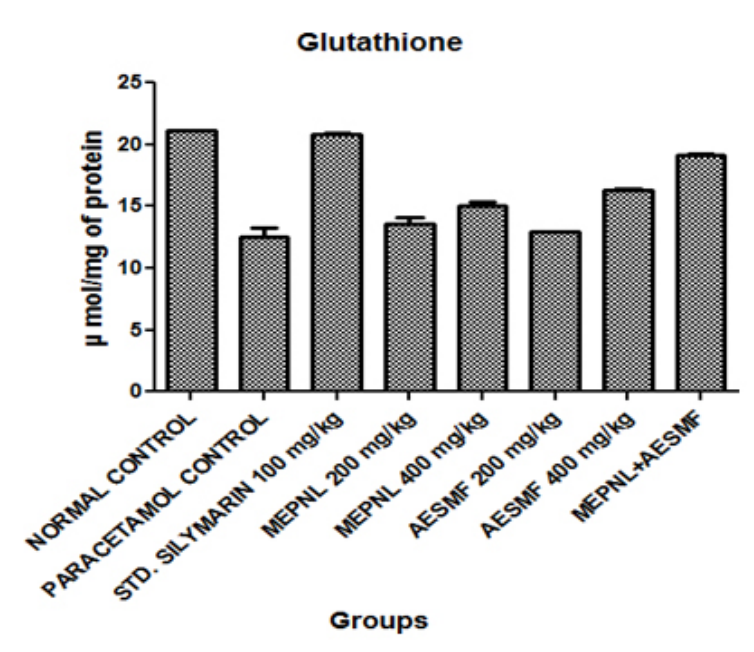

Figure 11: Effect of MEPNL, AESMF and Combined extract (MEPNL+AESMF) on GSH in acetaminophen treated rats.

\section{DISCUSSION}

The above results indicate that the combined extract of Phyllanthus niruri leaves and Sapindus mukorossi fruit (MEPNL + AESMF) have exhibited remarkable antioxidant effect by scavenging the DPPH, NO, Hydrogen peroxide and $\mathrm{Su}-$ peroxide anion radicals. The extract mixture also increased reducing power activity and inhibition of bleaching of $\beta$-Carotene linoleic acid. The in vitro antioxidant potential of the combined extract is more than the antioxidant effects of individual extracts. In vivo, antioxidant results indicate that the extract mixture at $400 \mathrm{mg} / \mathrm{kg}$ inhibited lipid peroxidation more than the individual extracts and enhanced significantly the antioxidant enzymes such as Catalase, SOD and Glutathione greater than the individual extracts in albino rats. This signifies that the combined extract of Phyllanthus niruri leaves and Sapindus mukorossi fruit (MEPNL + AESMF) have synergistic antioxidant potentials. Various antioxidants follow various components or pathways under different stress conditions to show their antioxidative response. The principle systems include: hindering free radical oxidation responses (preventive oxidants), interference of propagation of the autoxidation chain response (chain-breaking antioxidants); inhibiting the formation of free lipid radicals, quenching single oxygen species; diminishing hydroperoxides changing over them into stable compounds; inhibiting prooxidative enzymes; chelating metals and changing over metalpro-oxidants into stable products and through synergism with other antioxidants ${ }^{23,24}$.

\section{CONCLUSIONS}

From the above studies, we can conclude that the combined extract of Phyllanthus niruri leaves and Sapindus mukorossi fruit (MEPNL + AESMF) have potent and synergistic antioxidant activity. The antioxidant efficacy of the combined extract was found to be higher than the individual extracts. The exact mechanism behind this synergistic effect is not clearly understood but it can be hypothesized that the synergistic effect of flavonoids, saponins and other antioxidant components in Phyllanthus niruri leaves and Sapindus mukorossi fruits when used in combination can effectively neutralize free radicals that are responsible for the oxidative stress.

\section{ACKNOWLEDGEMENTS}

The authors are also thankful to Dr. N. Sivaraj, Senior Scientist (Eco Botany), National Bureau of Plant Genetic Resources, Rajendranagar, Hyderabad for kind co-operation in the authentication of plants. We would like to thanks to Institutional Animal Ethics Committee of Pharmacy College for providing ethical clearance of in vivo study for the present research.

\section{Source of Funding}

No source of funding

\section{Conflict of Interests}

This is to inform you that the authors declare that they have no conflict of interest regarding this article.

\section{Author Contribution}

Mrs. Nazia Malik has selected the plants and performed the activities under the supervision of Rakesh Kumar Jat and Darna Bhikshapathi.

\section{REFERENCES}

1. Abdel-Aziz SM, Aeron A, Kahil TA. Health benefits and possible risks of herbal medicine. Micr Food Health 2016; 97-116.

2. Zhao J. Phytonutrient and Phytotherapy for Improving Health. Aesthetic Med. 2012; 47-58.

3. Prior R, Cao G. Antioxidant Capacity and Polyphone Compounds of Teas. PSEBM 1999; 220: 255-261.

4. Azab AE, Albasha MO, Elsayed AS. Prevention of nephropathy by some natural sources of antioxidants. Yangtze Med 2017; 1(04):235.

5. Robinson EE, Maxwell SR, Thorpe GH. An investigation of the antioxidant activity of black tea using enhanced chemiluminescence. Free Rad Res. 1997; 26(3):291-302.

6. Fetouh FA, Azab AE. Ameliorating effects of curcumin and propolis against the reproductive toxicity of gentamicin in adult male Guinea pigs: Quantitative analysis and morphological study. Amer J Life Sci. 2014; 2(3):138-149.

7. Azab AE, Albasha MO. Hepatoprotective effect of some medicinal plants and herbs against hepatic disorders induced by hepatotoxic agents. J Biotechnol Bioeng. 2018;2(1):8-23.

8. Yin X, Zhou J, Jie C, Xing D, Zhang Y. Anticancer activity and mechanism of Scutellaria barbata extract on human lung cancer cell line A549. Life sci. 2004; 75(18):2233-44. 
9. Devita VT, Young RC, Canellos GP. Combination versus singleagent chemotherapy: a review of the basis for selection of drug treatment of cancer. Cancer 1975; 35: 98- 110.

10. Liu J, Huang YM, Wang H. Research progress of network pharmacology. West China J Pharm Sci. 2014; 29:723-725.

11. Manonmani P, Ramar M, Geetha N, Arasu MV, Erusan RR, Sowmiya JJ. Hepatoprotective activity of aqueous extract of Phyllanthus niruri in $\mathrm{CCl} 4$ induced liver toxicity-in vivo study. Res J Biotech. 2015;10:9.

12. The Useful Plants of India. Publication and Information Directorate. New Delhi;1986.p.547-553.

13. Nikunj B, Varsha J, Bharatkumar G.Antistress activity of Argyreia speciosa roots in experimental animals. J Ayurveda Integr Med. 2011; 2(3): 129-136.

14. Gutierrez RMP, Navarro YTG. Antioxidant and hepatoprotective effects of the methanol extract of the leaves of Satureja macrostema.Pharmacog Mag 2010;6(22):125-31.

15. Oyaizu M. Studies on products of the browning reaction. Jap J Nutr Diet .1986; 44(6):307-15.

16. Ruch RJ, Cheng SJ, Klaunig JE. Prevention of cytotoxicity and inhibition of intercellular communication by antioxidant catechins isolated from Chinese green tea. Carcinog. 1989;10(6):1003-8.
17. Kilicgun H, Altiner D.Correlation between antioxidant effect mechanisms and polyphenol content of Rosa canina. Pharmacog Mag. 2010; 6(23):238-41.

18. Khanam S, Shivprasad N, Devi K.Natural Antimutagens. Ind J Pharm Educ. 2004; 38(4): 180- 83

19. OECD Guidelines, OECD guidelines for testing of chemicals, Test No. 425: Acute oral toxic. 1996.

20. Ballantyne B, Timothy M, Turner P. General and Applied Toxicology, Adbridge Ed., The Macmillan press limited, London; 1995;53-56.

21. Krishna KL, Mruthunjaya K, Jagruthi A. Antioxidant and Hepatoprotective Potential of Stem Methanolic Extract of Justicia gendarussa. Burm Inter J Pharmacol. 2010; 6 (2):72-80.

22. Setty SR, Swamy AHM, Patil T, Prakash T, Gouda A V.Evaluation of hepatoprotective and in-vitro cytotoxic activity of leaves of Chrozophora plicata Linn. Fitoterapia. 2007; 79:51-54.

23. Kancheva VD. Phenolic antioxidants - radical-scavenging and chain-breaking activity: a comparative study. Eur J Lipid Sci Technol. 2009; 111:1072-1089.

24. Singh M, Pragzna Y, Dharmadev B. In-vitro Estimation of Free Radical Scavenging Activity of Fruit juices by DPPH assay. Int J Curr Res Rev. 2012;17(4):67-73. 